Raúl Montes-De-Oca (México)

Alexander Sakhanenko (Puebla)

Francisco Salem-Silva (Puebla)

\title{
ESTIMATES FOR PERTURBATIONS OF DISCOUNTED MARKOV CHAINS ON GENERAL SPACES
}

Abstract. We analyse a Markov chain and perturbations of the transition probability and the one-step cost function (possibly unbounded) defined on it. Under certain conditions, of Lyapunov and Harris type, we obtain new estimates of the effects of such perturbations via an index of perturbations, defined as the difference of the total expected discounted costs between the original Markov chain and the perturbed one. We provide an example which illustrates our analysis.

1. Introduction. Suppose that we have two Markov chains $\Phi=\left\{\Phi_{n}\right\}$ and $\widetilde{\Phi}=\left\{\widetilde{\Phi}_{n}\right\}, n=0,1,2, \ldots$, taking their values in the same arbitrary measurable space $(\mathbb{X}, \mathcal{B})$. For some nonnegative measurable functions $c$ : $\mathbb{X} \rightarrow \mathbb{R}$ and $\widetilde{c}: \mathbb{X} \rightarrow \mathbb{R}$ we define the values

$$
V_{\alpha}(x)=\sum_{n=0}^{\infty} \alpha^{n} \mathbf{E}\left\{c\left(\Phi_{n}\right) \mid \Phi_{0}=x\right\}, \quad \widetilde{V}_{\alpha}(x)=\sum_{n=0}^{\infty} \alpha^{n} \mathbf{E}\left\{\widetilde{c}\left(\widetilde{\Phi}_{n}\right) \mid \widetilde{\Phi}_{0}=x\right\},
$$

where $0<\alpha<1$ and $x \in \mathbb{X}$. Our main goal is to estimate from above the differences

$$
\Delta_{\alpha}(x)=V_{\alpha}(x)-\widetilde{V}_{\alpha}(x) \quad \text { and } \quad \widetilde{\Delta}_{\alpha}(x)=\widetilde{V}_{\alpha}(x)-V_{\alpha}(x)
$$

when the functions $c(\cdot), \widetilde{c}(\cdot)$ and the kernels

$$
\mathbf{Q}(A \mid x)=\mathbf{P}\left(\Phi_{n+1} \in A \mid \Phi_{n}=x\right), \quad \widetilde{\mathbf{Q}}(A \mid x)=\mathbf{P}\left(\widetilde{\Phi}_{n+1} \in A \mid \widetilde{\Phi}_{n}=x\right)
$$

are close in some sense, where $x \in \mathbb{X}, A \in \mathcal{B}$ and $n=0,1,2, \ldots$

2000 Mathematics Subject Classification: 60J10, 93D05, 93D09.

Key words and phrases: Harris Markov chains, total discounted expected cost, index of perturbations, Lyapunov condition.

Research of F. Salem-Silva supported by grant VIEP - BUAP II 33 G01. 
This problem has the following intuitive background. Suppose that $c(z)$ is the cost that the chain $\Phi$ pays at time $t$ when $\Phi_{t}=z$. In this case the volume $V_{\alpha}(x)$ may be called the total expected $\alpha$-discounted cost when $\Phi_{0}=x$. But in practice the functions $c(\cdot)$ and $\mathbf{Q}(\cdot \cdot \cdot)$ are partially known, because generally they are obtained by measurement or by statistical estimation, making it impossible to know $V_{\alpha}(x)$ completely. In this situation we can suppose that we have another two known functions $\widetilde{c}(\cdot)$ and $\widetilde{\mathbf{Q}}(\cdot \mid \cdot)$ and some information about the differences

$$
c(\cdot)-\widetilde{c}(\cdot) \quad \text { and } \operatorname{dist}(\mathbf{Q}(\cdot \mid \cdot), \widetilde{\mathbf{Q}}(\cdot \cdot \cdot))
$$

where $\operatorname{dist}(\cdot, \cdot)$ is some probabilistic metric.

And now we want to estimate the value $V_{\alpha}(x)$ using this information. It is clear that it is sufficient to obtain the estimates from above for the values $\Delta_{\alpha}(x)$ and $\widetilde{\Delta}_{\alpha}(x)$, defined in $(2)$.

An analogous, but more difficult, problem is known for Markov control processes. We mean the problem of obtaining estimates for the so-called "stability index" or "index of perturbations" (see, for example [3]-[6], [11] and the references there). But, really, the main difficulty in the proofs of the above-mentioned results consists in obtaining estimates for differences (2) for two fixed Markov chains without any control. For this reason, the differences $\Delta_{\alpha}(x)$ and $\widetilde{\Delta}_{\alpha}(x)$ may be called indices of perturbations.

In this paper we investigate the problem stated above without "noising control assumption". Our aim is to obtain estimates which should contain only explicitly defined functions and constants, and should have simple proofs (see Theorem 1 and Corollary 2 below).

We emphasize that we consider Markov chains taking their values in an arbitrary space. For countable Markov chains several estimates in similar problems were considered earlier in [1] and [2].

The main estimates are presented in Section 2 and their proofs are gathered in Section 3. In Section 4 we give an example that satisfies our conditions.

\section{Main results}

2.1. Notations. We consider kernels

$$
\mathbf{Q}(A \mid x), \quad \widetilde{\mathbf{Q}}(A \mid x) \quad \text { and } \quad \mathbf{K}(A \mid x), \quad x \in \mathbb{X}, A \in \mathcal{B},
$$

defined on an arbitrary space $\mathbb{X}$ with an arbitrary $\sigma$-algebra $\mathcal{B}$ and such that

$$
\forall x \quad \mathbf{Q}(\mathbb{X} \mid x)=\widetilde{\mathbf{Q}}(\mathbb{X} \mid x)=1 \quad \text { and } \quad \mathbf{K}(\mathbb{X} \mid x) \leq 1 .
$$

We stress that all the notations and conventions which we introduce in subsections 2.1 and 2.2 for the kernel $\mathbf{K}(\cdot \mid \cdot)$ will be used later on also for the kernels $\mathbf{Q}(\cdot \mid \cdot)$ and $\widetilde{\mathbf{Q}}(\cdot \mid \cdot)$. In these cases we will use the symbols $\mathbf{Q}$ or $\widetilde{\mathbf{Q}}$, respectively, instead of $\mathbf{K}$. 
We use the standard notations

$$
\mathbf{K}^{n}(\cdot \mid x)=\int \mathbf{K}^{n-1}(\cdot \mid y) \mathbf{K}(d y \mid x), \quad n=1,2, \ldots
$$

where $\mathbf{K}^{0}(A \mid x) \equiv I_{A}(x)$ is the indicator of the set $A$. Let

$$
\mathbf{K}_{\alpha}(A \mid x)=\sum_{n=0}^{\infty} \alpha^{n} \mathbf{K}^{n}(A \mid x), \quad x \in \mathbb{X}, A \in \mathcal{B}, 0<\alpha<1 .
$$

We define

$$
\mathbf{K}^{n} f(x)=\int f(y) \mathbf{K}^{n}(d y \mid x), \quad \mathbf{K}_{\alpha} f(x)=\sum_{n=0}^{\infty} \alpha^{n} \mathbf{K}^{n} f(x)
$$

for any function $f$ such that

$$
\forall x \quad \mathbf{K}_{\alpha} f^{+}(x)<\infty \quad \text { or } \quad \forall x \quad \mathbf{K}_{\alpha} f^{-}(x)<\infty,
$$

where we use the standard notations $a^{+}=\max \{a, 0\}$ and $a^{-}=\max \{-a, 0\}$ for positive and negative parts of a number. If $\mathbf{K}_{\alpha} f(\cdot)$ is defined, then it satisfies the equality

$$
\forall x \quad \mathbf{K}_{\alpha} f(x)=f(x)+\alpha \int \mathbf{K}_{\alpha} f(y) \mathbf{K}(d y \mid x) .
$$

Note that

$$
\forall x \quad\left|\mathbf{K}_{\alpha} f(x)\right|<\infty \quad \text { iff } \quad \forall x \quad \mathbf{K}_{\alpha}|f|(x)<\infty .
$$

For any measure $\boldsymbol{\mu}$ we use the notations

$$
\boldsymbol{\mu} f=\int f(x) \boldsymbol{\mu}(d x), \quad \boldsymbol{\mu} \mathbf{K}_{\alpha} f=\int \mathbf{K}_{\alpha} f(x) \boldsymbol{\mu}(d x) .
$$

If $\boldsymbol{\mu}$ and $\widetilde{\boldsymbol{\mu}}$ are two measures then $|\boldsymbol{\mu}-\widetilde{\boldsymbol{\mu}}|$ denotes the total variation of the signed measure $\boldsymbol{\mu}-\tilde{\boldsymbol{\mu}}$. For any function $W \geq 0$ we set

$$
\varrho_{W, m}(x)=\int W(x)\left|\mathbf{Q}^{m}(d y \mid x)-\widetilde{\mathbf{Q}}^{m}(d y \mid x)\right| .
$$

For functions $W(\cdot) \geq 0$ and $V(\cdot) \geq 0$ we use the norms

$$
\|f\|_{W}=\sup _{x \in \mathbb{X}} \frac{|f(x)|}{W(x)}, \quad\|f\|_{V}=\sup _{x \in \mathbb{X}} \frac{|f(x)|}{V(x)}, \quad\|f\|_{1+V}=\sup _{x \in \mathbb{X}} \frac{|f(x)|}{1+V(x)} .
$$

Here and everywhere in the paper we assume that $0 / 0=0$ and that $0 / c=\infty$ for $c>0$.

2.2. Special classes of kernels. We write $\mathbf{K}(\cdot \mid \cdot) \in \mathcal{L}(V, \lambda, b)$ if the function $V$ and the real numbers $\lambda, b$ are such that

$$
\begin{gathered}
\forall x \quad 0 \leq V(x)<\infty, \quad \mathbf{K} V(x) \leq \lambda V(x)+b, \quad \mathbf{K}(\mathbb{X} \mid x) \leq 1, \\
0 \leq \lambda<\infty, \quad 0 \leq b<\infty .
\end{gathered}
$$

The function $V(\cdot)$ is usually called a Lyapunov function. Assumptions of this type are used very often. See [4-6] and [7-10], for example. 
We write $\mathbf{K}(\cdot \mid \cdot) \in \mathcal{H}_{m}(W, \beta, w, \boldsymbol{\nu}, h)$ if there exist a probability measure $\nu$, functions $W$ and $h$, real numbers $\beta$ and $w$ and an integer $m \geq 1$ such that:

$$
\begin{aligned}
& \forall x \in \mathbb{X} \forall A \in \mathcal{B} \quad \mathbf{K}^{m}(A \mid x) \geq h(x) \boldsymbol{\nu}(A) \geq 0, \\
& \int W(y) \mathbf{K}^{m}(d y \mid x) \leq \beta W(x)+h(x) \int W(y) \boldsymbol{\nu}(d y)<\infty, \\
& 0 \leq \beta<\infty, \quad \int W(y) \boldsymbol{\nu}(d y) \leq w<\infty, \\
& \forall x \in \mathbb{X} \quad 0 \leq h(x) \leq 1 \leq W(x)<\infty, \quad \mathbf{K}(\mathbb{X} \mid x)=1 .
\end{aligned}
$$

REMARK 1. It is easy to see that the first assumption in (8) is a part of a Harris type condition. For example, if $h(x)=$ const $\cdot I_{C}(x)$, where $I_{C}(\cdot)$ is the indicator of the set $C$, then $C$ is frequently called ([10]) a small set. Thus, assumptions (8) may be considered as a natural combination of the Lyapunov and Harris conditions.

Assumptions (8) were used, for example, in the papers [1, 9], but with the additional restrictions on the value $\int h(y) \boldsymbol{\nu}(d y)$.

We write $\mathbf{K}(\cdot \mid \cdot) \in \mathcal{H}_{m}(W, \beta, w)$ if there exist a probability measure $\boldsymbol{\nu}$ and a function $h$ such that $\mathbf{K}(\cdot \mid \cdot) \in \mathcal{H}_{m}(W, \beta, w, \boldsymbol{\nu}, h)$. It is easy to see that

$$
\mathcal{H}_{1}(V(\cdot), \lambda, b) \subset \mathcal{L}(V(\cdot), \lambda, b) \subset \mathcal{L}(1+V(\cdot), \lambda, b+1-\lambda) .
$$

2.3. Key estimates. Suppose that a kernel $\mathbf{Q}(\cdot \mid \cdot)$ and a function $c(\cdot)$ satisfy

$$
\forall x \quad\left|\mathbf{Q}_{\alpha} c(x)-C_{0}\right| \leq C_{1} W(x)<\infty
$$

with some function $W(\cdot) \geq 0$ and constants $C_{0}$ and $C_{1}$. Introduce also the following assumptions:

$$
\begin{gathered}
\widetilde{\mathbf{Q}}(\cdot \mid \cdot) \in \mathcal{L}(V, \lambda, b), \quad 0 \leq \alpha \lambda<1, \quad 0 \leq \alpha<1, \\
\|\widetilde{c}(\cdot)\|_{1+V}<\infty, \quad\|W(\cdot)\|_{1+V}<\infty .
\end{gathered}
$$

Theorem 1. Assume that conditions (3) and (9)-(11) are satisfied. Then

$$
\begin{aligned}
& \Delta_{\alpha}(x):=\mathbf{Q}_{\alpha} c(x)-\widetilde{\mathbf{Q}}_{\alpha} \widetilde{c}(x) \leq\left(V(x)+\frac{\alpha b}{1-\alpha}\right) \frac{\delta_{1}^{(+)}}{1-\alpha \lambda}, \\
& \widetilde{\Delta}_{\alpha}(x):=\widetilde{\mathbf{Q}}_{\alpha} \widetilde{c}(x)-\mathbf{Q}_{\alpha} c(x) \leq\left(V(x)+\frac{\alpha b}{1-\alpha}\right) \frac{\delta_{1}^{(-)}}{1-\alpha \lambda},
\end{aligned}
$$

for all $x \in \mathbb{X}$, where

$$
\delta_{1}^{( \pm)}=\left\|(\widetilde{c}-c)^{ \pm}\right\|_{V}+\alpha C_{1}\left\|\varrho_{W, 1}\right\|_{V} .
$$

Thus, if we want to apply Theorem 1 we need first to obtain simple sufficient conditions for assumption (9) to be satisfied. The simplest such condition may be found in the following 
Theorem 2. Suppose that

$$
\mathbf{Q}(\cdot \mid \cdot) \in \mathcal{H}_{1}(W, \beta, w, \nu, h) \quad \text { and } \quad\|c\|_{W}<\infty .
$$

Then inequality (9) is true with

$$
C_{0}=\nu \mathbf{Q}_{\alpha} c, \quad C_{1}=\frac{\left\|c(\cdot)-(1-\alpha) C_{0}\right\|_{W}}{1-\alpha \beta}, \quad(1-\alpha) C_{0} \leq \frac{w\|c\|_{W}}{1-\alpha \beta} .
$$

2.4. Now we may obtain several simple useful corollaries.

Corollary 1. Assume that

$$
\mathbf{Q}(\cdot \mid \cdot) \in \mathcal{H}_{1}(W, \beta, w), \quad\|c(\cdot)\|_{W}<\infty, \quad 0 \leq \alpha \beta<1,
$$

and that conditions (3), (10) and (11) are satisfied. Then inequalities (12) and (13) hold for all $x \in \mathbb{X}$ with $C_{1}$ defined in (16). In particular

$$
C_{1} \leq \frac{(w+1-\alpha \beta)}{(1-\alpha \beta)^{2}}\|c\|_{W} .
$$

This assertion follows immediately from Theorems 1 and 2 .

Corollary 2. Assume that condition (3) is satisfied and that

$$
\begin{gathered}
\mathbf{Q}(\cdot \mid \cdot) \in \mathcal{H}_{1}(W, \beta, w), \quad \widetilde{\mathbf{Q}}(\cdot \mid \cdot) \in \mathcal{H}_{1}(W, \beta, w), \\
0 \leq \alpha \beta<1, \quad 0 \leq \alpha<1, \quad\|c(\cdot)\|_{W}<\infty, \quad\|\widetilde{c}(\cdot)\|_{W}<\infty .
\end{gathered}
$$

Then, for all $x \in \mathbb{X}$,

$$
\begin{aligned}
& \Delta_{\alpha}(x) \leq\left(W(x)+\frac{\alpha w}{1-\alpha}\right)\left(\frac{\left\|(c-\widetilde{c})^{+}\right\|_{W}}{1-\alpha \beta}+\frac{C_{2}\left\|\varrho_{W, 1}\right\|_{W}}{(1-\alpha \beta)^{3}}\right), \\
& \widetilde{\Delta}_{\alpha}(x) \leq\left(W(x)+\frac{\alpha w}{1-\alpha}\right)\left(\frac{\left\|(\widetilde{c}-c)^{+}\right\|_{W}}{1-\alpha \beta}+\frac{C_{2}\left\|\varrho_{W, 1}\right\|_{W}}{(1-\alpha \beta)^{3}}\right),
\end{aligned}
$$

where

$$
C_{2}=\alpha(w+1-\alpha \beta) \min \left\{\|c\|_{W},\|\widetilde{c}\|_{W}\right\} .
$$

To prove this assertion we need to apply Corollary 1 with $\lambda:=\beta$ and $b:=w$. But if $\|c(\cdot)\|_{W}>\|\widetilde{c}(\cdot)\|_{W}$ then we must repeat these arguments using the values $\widetilde{\mathbf{Q}}$ and $\widetilde{c}$ instead of $\mathbf{Q}$ and $c$, and vice versa.

2.5. Case $m>1$. This case is a little more complicated. We need the following additional notation:

$$
\mathbf{Q}_{\alpha, m} f(x)=\sum_{n=0}^{\infty} \alpha^{m n} \mathbf{Q}^{m n} f(x), \quad m=1,2, \ldots,
$$

which may be used for any function $f$ such that

$$
\forall x \quad \mathbf{Q}_{\alpha} f^{+}(x)<\infty \quad \text { or } \quad \forall x \quad \mathbf{Q}_{\alpha} f^{-}(x)<\infty .
$$


Suppose now that a kernel $\mathbf{Q}(\cdot \mid \cdot)$ and a function $c(\cdot)$ satisfy

$$
\forall x \quad\left|\mathbf{Q}_{\alpha, m} c(x)-C_{0, m}\right| \leq C_{1, m} W(x)<\infty
$$

with some function $W(\cdot) \geq 0$, integer $m$ and constants $C_{0, m}$ and $C_{1, m}$.

Theorem 3. Assume that conditions (3), (10), (11) and (18) are satisfied for some $m>1$. Then, for all $x \in \mathbb{X}$,

$$
\begin{aligned}
& \Delta_{\alpha}(x) \leq\left(V(x)+\frac{\alpha b}{1-\alpha}\right) \frac{\delta_{m}^{(+)}}{1-\alpha \lambda}+\delta_{m, \alpha}(x), \\
& \widetilde{\Delta}_{\alpha}(x) \leq\left(V(x)+\frac{\alpha b}{1-\alpha}\right) \frac{\delta_{m}^{(-)}}{1-\alpha \lambda}+\delta_{m, \alpha}(x),
\end{aligned}
$$

where

$$
\delta_{m}^{( \pm)}=\left\|(\widetilde{c}-c)^{ \pm}\right\|_{V}+\alpha C_{1, m}\left\|\varrho_{W, m}\right\|_{V}, \quad \delta_{m, \alpha}(x)=C_{1, m} \sum_{k=1}^{m-1} \alpha^{k} \varrho_{W, k}(x) .
$$

The simplest sufficient condition for (18) to hold may be found in the following

Theorem 4. Suppose that

$$
\mathbf{Q}(\cdot \mid \cdot) \in \mathcal{H}_{m}(W, \beta, w, \nu, h) \quad \text { and } \quad\|c\|_{W}<\infty .
$$

Then inequality (18) is true with

$$
\begin{aligned}
C_{0, m} & =\boldsymbol{\nu} \mathbf{Q}_{\alpha, m} c, \quad\left(1-\alpha^{m}\right) C_{0, m} \leq \frac{w\|c\|_{W}}{1-\alpha^{m} \beta}, \\
C_{1, m} & =\frac{\left\|c(\cdot)-\left(1-\alpha^{m}\right) C_{0, m}\right\|_{W}}{1-\alpha^{m} \beta} \leq \frac{w+1-\alpha^{m} \beta}{\left(1-\alpha^{m} \beta\right)^{2}}\|c\|_{W} .
\end{aligned}
$$

The following simple corollary follows immediately from Theorems 3 and 4 .

Corollary 3. Assume that

$$
\mathbf{Q}(\cdot \mid \cdot) \in \mathcal{H}_{m}(W, \beta, w), \quad\|c(\cdot)\|_{W}<\infty, \quad 0 \leq \alpha^{m} \beta<1,
$$

and that conditions (3), (10) and (11) are satisfied. Then inequalities (19) and (20) hold for all $x \in \mathbb{X}$ with $C_{1}$ from (22).

REMARK 2. In this paper we consider only the case when the known kernel $\widetilde{\mathbf{Q}}(\cdot \mid \cdot)$ satisfies the Lyapunov type condition (10), whereas for the initial kernel $\mathbf{Q}(\cdot \mid \cdot)$ assumptions of another type are supposed to be valid. But we may apply all the assertions of the paper also in the case when $\widetilde{\mathbf{Q}}(\cdot \cdot \cdot)$ is the initial kernel that satisfies the Lyapunov type condition (10), whereas $\mathbf{Q}(\cdot \mid \cdot)$ is the known kernel for which the above-mentioned assumptions of another type are valid. 


\section{Proofs}

3.1. Auxiliary lemmas

Lemma 1 . If

$$
\mathbf{K}(\cdot \mid \cdot) \in \mathcal{L}(V, \lambda, b) \quad \text { and } \quad\left\|f^{+}\right\|_{V}<\infty
$$

then the function $\mathbf{K}_{\alpha} f(\cdot)$ is defined, satisfies (5) and

$$
\mathbf{K}^{n} f(x) \leq\left\|f^{+}\right\|_{V} \mathbf{K}^{n} V(x) \leq\left\|f^{+}\right\|_{V}\left(\lambda^{n} V(x)+\sum_{0 \leq k<n} b \lambda^{k}\right),
$$

for all $n=1,2, \ldots$ In addition, for all $\alpha \in(0, \min \{1,1 / \lambda\})$,

$$
\mathbf{K}_{\alpha} f(x) \leq\left\|f^{+}\right\|_{V} \mathbf{K}_{\alpha} V(x), \quad \mathbf{K}_{\alpha} f(x) \leq\left\|f^{+}\right\|_{1+V}\left(1+\mathbf{K}_{\alpha} V(x)\right),
$$

where

$$
\mathbf{K}_{\alpha} V(x)=\sum_{n=0}^{\infty} \alpha^{n} \mathbf{K}^{n} V(x) \leq\left(V(x)+\frac{\alpha b}{1-\alpha}\right) \frac{1}{1-\alpha \lambda} .
$$

It is not difficult to verify these inequalities immediately.

Lemma 2. Suppose that the kernel $\mathbf{K}(\cdot \mid \cdot)$ and functions $f$ and $F$ are such that

$$
\begin{aligned}
& \mathbf{K}(\cdot \mid \cdot) \in \mathcal{L}(V, \lambda, b), \quad\left\|f^{+}\right\|_{1+V}+\left\|F^{+}\right\|_{1+V}<\infty, \\
& \forall x \quad F(x) \leq f(x)+\alpha \int F(y) \mathbf{Q}(d y \mid x)
\end{aligned}
$$

and $\alpha \in(0, \min \{1,1 / \lambda\})$. Then

$$
\forall x \quad F(x) \leq \mathbf{K}_{\alpha} f^{+}(x) .
$$

Proof. Lemma 1 and (5) yield

$$
\forall x \quad \mathbf{K}_{\alpha} f^{+}(x)=f^{+}(x)+\alpha \int \mathbf{K}_{\alpha} f^{+}(y) \mathbf{K}(d y \mid x)<\infty .
$$

Hence, for

$$
\Delta(x):=F(x)-\mathbf{K}_{\alpha} f^{+}(x) \leq F(x) \leq\left\|F^{+}\right\|_{1+V}(1+V(x))
$$

we deduce from (25) and (27) that

$$
\Delta(x) \leq f(x)-f^{+}(x)+\alpha \int \Delta(y) \mathbf{K}(d y \mid x) .
$$

Thus for all $n=1,2,3, \ldots$,

$$
\forall x \quad \Delta(x) \leq \alpha \int \Delta(y) \mathbf{K}(d y \mid x) \leq \alpha^{n} \int \Delta(y) \mathbf{K}^{n}(d y \mid x) .
$$

Now (23), (28) and (29) yield

$$
\begin{aligned}
\Delta(x) & \leq\left\|F^{+}\right\|_{1+V} \cdot \alpha^{n} \int(1+V(y)) \mathbf{K}^{n}(d y \mid x) \\
& \leq\left\|F^{+}\right\|_{1+V}\left(\alpha^{n}+\alpha^{n} \lambda^{n} V(x)+\alpha^{n} \sum_{0 \leq k<n} b \lambda^{k}\right) \\
& \leq\left\|F^{+}\right\|_{1+V}\left(\alpha^{n}+\alpha^{n} \lambda^{n} V(x)+\alpha^{n} \cdot n b\left(1+\lambda^{n}\right)\right) \rightarrow 0
\end{aligned}
$$


as $n \rightarrow \infty$ because $\alpha \in(0,1)$ and $\alpha \lambda \in(0,1)$. Hence $\Delta(x) \leq 0$ for all $x$ and (26) follows from definition (28).

3.2. Proof of Theorem 1. We first prove inequality (12). We may assume that the right hand side in (12) is finite, that is,

$$
\left\|(\widetilde{c}-c)^{+}\right\|_{V}<\infty \text { and }\left\|\varrho_{W, 1}\right\|_{V}<\infty .
$$

We divide the proof of (12) into several lemmas.

Lemma 3. If conditions (9)-(11) are satisfied then

$$
\left\|\mathbf{Q}_{\alpha} c\right\|_{1+V}<\infty \text { and }\left\|\widetilde{\mathbf{Q}}_{\alpha} \widetilde{c}\right\|_{1+V}<\infty .
$$

In addition, the function $\widetilde{\mathbf{Q}} \mathbf{Q}_{\alpha} c(\cdot)$ is well defined and finite.

Proof. From (9) we have

$$
\forall x \quad\left|\mathbf{Q}_{\alpha} c(x)\right| \leq\left|C_{0}\right|+C_{1} W(x)
$$

and using (11) we obtain

$$
\left\|\mathbf{Q}_{\alpha} c\right\|_{1+V} \leq\left|C_{0}\right|+C_{1}\|W\|_{1+V}<\infty .
$$

The second inequality in (31) follows from (10), (11) and Lemma 1 with $\mathbf{K}(\cdot \mid \cdot):=\widetilde{\mathbf{Q}}(\cdot \mid \cdot)$. The same arguments give $\left\|\widetilde{\mathbf{Q}}\left|\mathbf{Q}_{\alpha} c\right|\right\|_{1+V}<\infty$. Hence, the function $\widetilde{\mathbf{Q}} \mathbf{Q}_{\alpha} c(x)$ exists and is finite.

By Lemma 3 we may introduce the following notation:

$$
J_{1}(x):=\int \mathbf{Q}_{\alpha} c(y) \mathbf{Q}(d y \mid x)-\int \mathbf{Q}_{\alpha} c(y) \widetilde{\mathbf{Q}}(d y \mid x) .
$$

LEMma 4. If conditions (3) and (9) are satisfied then

$$
\forall x \quad\left|J_{1}(x)\right| \leq C_{1} \varrho_{W, 1}(x) \quad \text { and } \quad\left\|J_{1}(x)\right\|_{V} \leq C_{1}\left\|\varrho_{W, 1}\right\|_{V} .
$$

Proof. Using (3) and (32) we obtain

$$
J_{1}(x)=\int\left(\mathbf{Q}_{\alpha} c(y)-C_{0}\right)(\mathbf{Q}(d y \mid x)-\widetilde{\mathbf{Q}}(d y \mid x))
$$

for any constant $C_{0}$. Hence, by (34), (9) and (6),

$$
\begin{aligned}
\forall x \quad\left|J_{1}(x)\right| & \leq \int\left|\mathbf{Q}_{\alpha} c(y)-C_{0}\right||\mathbf{Q}(d y \mid x)-\widetilde{\mathbf{Q}}(d y \mid x)| \\
& \leq C_{1} \int W(y)|\mathbf{Q}(d y \mid x)-\widetilde{\mathbf{Q}}(d y \mid x)|=C_{1} \varrho_{W, 1}(x) .
\end{aligned}
$$

It is easy to see that (35) implies (33).

Lemma 5. Suppose that (30) and all the conditions of Theorem 1 are satisfied. Then

$$
\forall x \quad \Delta(x):=\mathbf{Q}_{\alpha} c(x)-\widetilde{\mathbf{Q}}_{\alpha} \widetilde{c}(x) \leq \widetilde{\mathbf{Q}}_{\alpha} V(x) \delta_{1}^{(+)},
$$

where the value $\delta_{1}^{(+)}$was defined in (14). 
Proof. By (5),

$$
\begin{aligned}
\mathbf{Q}_{\alpha} c(x) & =c(x)+\alpha \int \mathbf{Q}_{\alpha} c(y) \mathbf{Q}(d y \mid x), \\
\widetilde{\mathbf{Q}}_{\alpha} \widetilde{c}(x) & =\widetilde{c}(x)+\alpha \int \widetilde{\mathbf{Q}}_{\alpha} \widetilde{c}(y) \widetilde{\mathbf{Q}}(d y \mid x) .
\end{aligned}
$$

From (36)-(38) we obtain

$$
\Delta(x)=c(x)-\widetilde{c}(x)+\alpha J(x),
$$

where

$$
J(x)=\int \mathbf{Q}_{\alpha} c(y) \mathbf{Q}(d y \mid x)-\int \widetilde{\mathbf{Q}}_{\alpha} \widetilde{c}(x) \widetilde{\mathbf{Q}}(d y \mid x) \equiv J_{1}(x)+J_{2}(x) .
$$

From (32) and (40) we have

$$
J_{2}(x):=\int\left(\mathbf{Q}_{\alpha} c(y)-\widetilde{\mathbf{Q}}_{\alpha} \widetilde{c}(y)\right) \widetilde{\mathbf{Q}}(d y \mid x) \equiv \int \Delta(y) \widetilde{\mathbf{Q}}(d y \mid x) .
$$

So, using (39)-(41) we obtain

$$
\Delta(x)=f(x)+\alpha \int \Delta(y) \widetilde{\mathbf{Q}}(d y \mid x)
$$

with

$$
f(x)=c(x)-\widetilde{c}(x)+\alpha J_{1}(x) .
$$

It follows from (30), (33) and (43) that

$$
\left\|f^{+}\right\|_{1+V} \leq\left\|f^{+}\right\|_{V} \leq\left\|(\widetilde{c}-c)^{+}\right\|_{V}+\left\|\varrho_{W, 1}\right\|_{V}<\infty .
$$

By (36) and Lemma 3,

$$
\|\Delta(\cdot)\|_{1+V} \leq\left\|\widetilde{\mathbf{Q}}_{\alpha} \widetilde{c}\right\|_{1+V}+\left\|\mathbf{Q}_{\alpha} c\right\|_{1+V}<\infty .
$$

From (42)-(45) we see that we may apply Lemma 2 with $F(x):=\Delta(x)$ and $\mathbf{K}(\cdot \mid \cdot):=\widetilde{\mathbf{Q}}(\cdot \mid \cdot)$ to obtain

$$
\forall x \quad \Delta(x) \leq \widetilde{\mathbf{Q}}_{\alpha}(\widetilde{c}-c)^{+}(x)+\alpha \widetilde{\mathbf{Q}}_{\alpha} J_{1}^{+}(x) .
$$

It is easy to see that (46) and Lemma 1 with $\mathbf{K}(\cdot \mid \cdot):=\widetilde{\mathbf{Q}}(\cdot \mid \cdot)$ yield

$$
\forall x \quad \Delta(x) \leq \widetilde{\mathbf{Q}}_{\alpha} V(x)\left(\left\|(c-\widetilde{c})^{+}\right\|_{V}+\alpha\left\|J_{1}\right\|_{V}\right) .
$$

Now (36) follows from (33) and (47).

The desired inequality (12) is an immediate corollary of (36) and (24).

Thus inequality (12) is proved. To obtain the last assertion (13) of Theorem 1 , we only need to change in (12) the functions $c$ and $\widetilde{c}$ into $-c$ and $-\widetilde{c}$, respectively.

3.3. Proof of Theorem 2. Suppose that all the assumptions of Theorem 2 are satisfied. Define

$$
\mathbf{K}(A \mid x)=\mathbf{Q}(A \mid x)-h(x) \boldsymbol{\nu}(A) \geq 0, \quad x \in \mathbb{X}, A \in \mathcal{B} .
$$

Note that

$$
\mathbf{Q}(\cdot \mid \cdot) \in \mathcal{L}(W, \beta, w) \quad \text { and } \quad \mathbf{K}(\cdot \mid \cdot) \in \mathcal{L}(W, \beta, 0)
$$


as follows from (15) and the definitions of the classes $\mathcal{L}$ and $\mathcal{H}$. Let

$$
V_{0}(x)=\mathbf{Q}_{\alpha} c(x)-C_{0} \quad \text { and } \quad c_{0}(x)=c(x)-(1-\alpha) C_{0},
$$

where $C_{0}$ is defined in (16). It follows from (37) and (50) that

$$
V_{0}(x)=c_{0}(x)+\alpha \int V_{0}(y) \mathbf{Q}(d y \mid x) .
$$

Using the definitions (48) and (16) it is easy to verify that

$$
\begin{aligned}
& \int V_{0}(y) \mathbf{Q}(d y \mid x)-\int V_{0}(y) \mathbf{K}(d y \mid x)=h(x) \int V_{0}(y) \boldsymbol{\nu}(d y) \\
&=h(x)\left(\int \mathbf{Q}_{\alpha} c(y) \boldsymbol{\nu}(d y)-C_{0}\right)=h(x)\left(\boldsymbol{\nu} \mathbf{Q}_{\alpha} c-\boldsymbol{\nu} \mathbf{Q}_{\alpha} c\right)=0 .
\end{aligned}
$$

Thus, from (34) and (52) we have

$$
V_{0}(x)=c_{0}(x)+\alpha \int V_{0}(y) \mathbf{K}(d y \mid x) .
$$

It follows immediately from (49) and (53) that we may apply Lemma 2 with $F(\cdot):=V_{0}(\cdot), f(\cdot):=c_{0}(\cdot), \lambda:=\beta, b:=0$ and $V(\cdot):=W(\cdot)$. We have $V_{0}(x)=\mathbf{K}_{\alpha} c_{0}(x)$ and so

$$
\left\|V_{0}^{+}\right\|_{W} \leq(1-\alpha \beta)^{-1}\left\|c_{0}^{+}\right\|_{W} .
$$

Using (54) with $-c_{0}$ instead of $c_{0}$, we obtain

$$
\left\|V_{0}^{-}\right\|_{W} \leq(1-\alpha \beta)^{-1}\left\|c_{0}^{-}\right\|_{W} .
$$

Note that (9) is a special case of (54) and (55), by definition (50).

3.4. Proof of Theorem 3. We first prove (19). We may assume that the right hand side of (19) is finite, that is,

$$
\left\|(\widetilde{c}-c)^{+}\right\|_{V}<\infty \text { and }\left\|\varrho_{W, m}\right\|_{V}<\infty .
$$

We first prove several lemmas.

Lemma 6. If conditions (10), (11) and (18) are satisfied for some integer $m$, then

$$
\left\|\mathbf{Q}_{\alpha, m} c\right\|_{1+V}<\infty \quad \text { and }\left\|\widetilde{\mathbf{Q}}_{\alpha, m} \widetilde{c}\right\|_{1+V}<\infty .
$$

In addition, the functions $\widetilde{\mathbf{Q}}^{n} \mathbf{Q}_{\alpha, m} c(\cdot)$ are well defined and finite for all integers $n$.

Proof. Condition (10) and Lemma 1 yield

$$
\widetilde{\mathbf{Q}}^{n}(\cdot \mid \cdot) \in \mathcal{L}\left(V, \lambda^{n}, b_{n}\right) \quad \text { with } \quad b_{n}=\sum_{0 \leq k<n} b \lambda^{k}<\infty
$$

for all integers $n$. This allows us to repeat the proof of Lemma 3 with the kernels $\widetilde{\mathbf{Q}}^{n}, \widetilde{\mathbf{Q}}_{\alpha, m}$ and $\mathbf{Q}_{\alpha, m}$ instead of $\widetilde{\mathbf{Q}}, \widetilde{\mathbf{Q}}_{\alpha}$ and $\mathbf{Q}_{\alpha}$, respectively.

By Lemma 6 we may introduce the following notation:

$$
J_{m . n}(x):=\int \mathbf{Q}_{\alpha, m} c(y) \mathbf{Q}^{n}(d y \mid x)-\int \mathbf{Q}_{\alpha, m} c(y) \widetilde{\mathbf{Q}}^{n}(d y \mid x) .
$$


LEMMA 7. If conditions (3) and (18) are satisfied then

$\forall x \quad\left|J_{m . n}(x)\right| \leq C_{1, m} \varrho_{W, n}(x) \quad$ and $\quad\left\|J_{m . n}(x)\right\|_{V} \leq C_{1, m}\left\|\varrho_{W, n}\right\|_{V}$.

Proof. Repeating the proof of Lemma 4 we obtain

$$
J_{m . n}(x)=\int\left(\mathbf{Q}_{\alpha, m} c(y)-C_{0, m}\right)\left(\mathbf{Q}^{n}(d y \mid x)-\widetilde{\mathbf{Q}}^{n}(d y \mid x)\right)
$$

for any constant $C_{0, m}$. Hence,

$$
\begin{aligned}
\forall x \quad\left|J_{m . n}(x)\right| & \leq \int\left|\mathbf{Q}_{\alpha, m} c(y)-C_{0, m}\right|\left|\mathbf{Q}^{n}(d y \mid x)-\widetilde{\mathbf{Q}}^{n}(d y \mid x)\right| \\
& \leq C_{1, m} \int W(y)\left|\mathbf{Q}^{n}(d y \mid x)-\widetilde{\mathbf{Q}}^{n}(d y \mid x)\right|=C_{1, m} \varrho_{W, 1}(x) .
\end{aligned}
$$

Lemma 8. Suppose that assumption (56) and all the conditions of Theorem 3 are satisfied. Then

$$
\forall x \quad \Delta^{(m)}(x):=\mathbf{Q}_{\alpha, m} c(x)-\widetilde{\mathbf{Q}}_{\alpha, m} \widetilde{c}(x) \leq \widetilde{\mathbf{Q}}_{\alpha, m} V(x) \delta_{m}^{(+)},
$$

where the values $\delta_{m}^{(+)}$were defined in $(21)$.

It is easy to see that Lemma 5 is a special case of Lemma 8 for $m=1$. On the other hand, if we repeat the proof of Lemma 5 with the kernels $\widetilde{\mathbf{Q}}$ and $\mathbf{Q}$ replaced by $\widetilde{\mathbf{Q}}^{m}$ and $\mathbf{Q}^{m}$, respectively, then we immediately obtain Lemma 8.

Introduce two new kernels

$$
\mathbf{Q}_{\alpha, m}^{\prime}=\sum_{k=0}^{m-1} \alpha^{k} \mathbf{Q}^{k} \quad \text { and } \quad \mathbf{Q}_{\alpha, m}^{\prime}=\sum_{k=0}^{m-1} \alpha^{k} \widetilde{\mathbf{Q}}^{k} .
$$

By definitions (4), (17) and (59) it is easy to see that

$$
\mathbf{Q}_{\alpha} c(x)=\mathbf{Q}_{\alpha, m}^{\prime} \mathbf{Q}_{\alpha, m} c(x) \quad \text { and } \quad \widetilde{\mathbf{Q}}_{\alpha} \widetilde{c}(x)=\widetilde{\mathbf{Q}}_{\alpha, m}^{\prime} \widetilde{\mathbf{Q}}_{\alpha, m} \widetilde{c}(x) .
$$

Hence

$$
\mathbf{Q}_{\alpha} c(x)-\widetilde{\mathbf{Q}}_{\alpha} \widetilde{c}(x) \equiv J_{3}(x)+J_{4}(x)
$$

where

$$
\begin{aligned}
& J_{3}(x)=\mathbf{Q}_{\alpha, m}^{\prime} \mathbf{Q}_{\alpha, m} c(x)-\widetilde{\mathbf{Q}}_{\alpha, m}^{\prime} \mathbf{Q}_{\alpha, m} c(x), \\
& J_{4}(x)=\widetilde{\mathbf{Q}}_{\alpha, m}^{\prime} \mathbf{Q}_{\alpha, m} c(x)-\widetilde{\mathbf{Q}}_{\alpha, m}^{\prime} \widetilde{\mathbf{Q}}_{\alpha, m} \widetilde{c}(x) .
\end{aligned}
$$

Using (59) we may rewrite (62) in the following way:

$$
J_{3}(x)=\sum_{k=0}^{m-1} \alpha^{k}\left(\mathbf{Q}^{k}-\widetilde{\mathbf{Q}}^{k}\right) \mathbf{Q}_{\alpha, m} c(x)=\sum_{k=1}^{m-1} \alpha^{k}\left(\mathbf{Q}^{k}-\widetilde{\mathbf{Q}}^{k}\right) \mathbf{Q}_{\alpha, m} c(x),
$$

because $\mathbf{Q}^{0}(A \mid x) \equiv \widetilde{\mathbf{Q}}^{0}(A \mid x) \equiv I_{A}(x)$ is the indicator of the set $A$ by definition. From (57) and (64) we obtain

$$
J_{3}(x)=\sum_{k=1}^{m-1} \alpha^{k} J_{m, k}(x)
$$


So, by Lemma 7 ,

$$
\left|J_{3}(x)\right| \leq \sum_{k=1}^{m-1} \alpha^{k} C_{1, m} \varrho_{W, k}(x) \equiv \delta_{m, \alpha}(x) .
$$

Using (58) we infer from (63) that

$$
J_{4}(x)=\widetilde{\mathbf{Q}}_{\alpha, m}^{\prime} \Delta^{(m)}(x) \leq \widetilde{\mathbf{Q}}_{\alpha, m}^{\prime} \widetilde{\mathbf{Q}}_{\alpha, m} V(x) \delta_{m}^{(+)} .
$$

But (60) and (66) yield

$$
J_{4}(x) \leq \mathbf{Q}_{\alpha} V(x) \delta_{m}^{(+)} \leq\left(V(x)+\frac{\alpha b}{1-\alpha}\right) \frac{\delta_{m}^{(+)}}{1-\alpha \lambda} .
$$

The last inequality in (67) follows from (24).

The desired inequality (19) is an immediate consequence of (61), (65) and (67). To obtain (20) we only need to change in (19) the functions $c$ and $\widetilde{c}$ into $-c$ and $-\widetilde{c}$, respectively.

4. An example. In this section we present an example which is a special case of the example provided in Section 5 of [4] (see also [6]). The version that we give here satisfies all the assumptions of Corollary 2 .

Consider two chains, $\Phi$ and $\widetilde{\Phi}$, with the state space $\mathbb{X}=[0, \infty)$, defined by the following recursive equations:

$$
\begin{array}{lll}
\Phi_{0}=x, & \Phi_{n+1}=\left(\Phi_{n}+\zeta_{n}\right)^{+}, & n=0,1,2, \ldots, \\
\widetilde{\Phi}_{0}=x, & \widetilde{\Phi}_{n+1}=\left(\widetilde{\Phi}_{n}+\widetilde{\zeta}_{n}\right)^{+}, & n=0,1,2, \ldots,
\end{array}
$$

where $x \in \mathbb{X}$ is a given state. We suppose that each of the two sequences

$$
\zeta, \zeta_{1}, \zeta_{2}, \ldots \text { and } \widetilde{\zeta}, \widetilde{\zeta}_{1}, \widetilde{\zeta}_{2}, \ldots
$$

consists of independent and identically distributed random variables. In this case, for all $x \in \mathbb{X}$ and $B \in \mathcal{B}$ we may define the kernels

$$
\begin{aligned}
& \mathbf{Q}(B \mid x):=\mathbf{P}\left(\Phi_{n+1} \in B \mid \Phi_{n}=x\right)=\mathbf{P}\left((x+\zeta)^{+} \in B\right), \\
& \widetilde{\mathbf{Q}}(B \mid x):=\mathbf{P}\left(\widetilde{\Phi}_{n+1} \in B \mid \widetilde{\Phi}_{n}=x\right)=\mathbf{P}\left((x+\widetilde{\zeta})^{+} \in B\right),
\end{aligned}
$$

where $\mathcal{B}$ denotes the Borel $\sigma$-algebra of $\mathbb{X}=[0, \infty)$.

For all real $q>0$ set

$$
\begin{aligned}
& \beta_{q}:=\max \left\{\mathbf{E} e^{q \zeta}, \mathbf{E} e^{q \widetilde{\zeta}}\right\}, \\
& r_{q}:=\int_{-\infty}^{\infty} \max \left\{e^{q x}, 1\right\}|d \mathbf{P}(\zeta<x)-d \mathbf{P}(\widetilde{\zeta}<x)| .
\end{aligned}
$$

Remark 3. Assume that

$$
\begin{aligned}
& \mathbf{E} \zeta<0, \quad \mathbf{E} \widetilde{\zeta}<0, \\
& \mathbf{E} e^{\tau \zeta}<\infty, \quad \mathbf{E} e^{\tilde{\tau} \widetilde{\zeta}}<\infty,
\end{aligned}
$$


for some $\tau>0$ and $\widetilde{\tau}>0$. It is well known (see, for example [4, p. 232]), that in this case

$$
\exists q>0: \quad \beta_{q}<1 .
$$

On the other hand, we may obtain (71) also in the case where one or both of the expectations in (70) do not exist.

REMARK 4. Assume that the distributions of the random variables $\zeta$ and $\widetilde{\zeta}$ have densities $g$ and $\widetilde{g}$, respectively, with respect to some measure $\boldsymbol{\mu}$. It is easy to see that in this case we may calculate the value $r_{q}$ by the formula

$$
r_{q} \equiv \int_{-\infty}^{0}|g(x)-\widetilde{g}(x)| \boldsymbol{\mu}(d x)+\int_{0}^{\infty} e^{q x}|g(x)-\widetilde{g}(x)| \boldsymbol{\mu}(d x) .
$$

Proposition 1. Assume that the numbers $\alpha, q$ and $\beta_{q}$ are such that

$$
0 \leq \alpha<1, \quad 0 \leq \alpha \beta_{q}<1, \quad q>0 .
$$

Then the kernels $\mathbf{Q}(\cdot \mid \cdot)$ and $\widetilde{\mathbf{Q}}(\cdot \mid \cdot)$, introduced in (68), satisfy all the assumptions and assertions of Corollary 2 with

$$
W(x):=e^{q x}, \quad \beta:=\beta_{q}, \quad w:=1, \quad\left\|\varrho_{W, 1}\right\|_{W} \leq r_{q} .
$$

Suppose now that, in addition to assumptions (73),

$$
\forall x \quad c(x)=\widetilde{c}(x) \quad \text { and } \quad|c(x)| \leq d e^{q x} .
$$

In this case the assertions of Corollary 2 may by written in the following very simple form:

$$
\forall x \geq 0 \quad\left|V_{\alpha}(x)-\widetilde{V}_{\alpha}(x)\right| \leq r_{q} d \frac{\alpha\left(2-\alpha \beta_{q}\right)}{\left(1-\alpha \beta_{q}\right)^{3}}\left(e^{q x}+\frac{\alpha}{1-\alpha}\right),
$$

where the values $V_{\alpha}(\cdot)$ and $\widetilde{V}_{\alpha}(\cdot)$ were defined in (1).

The rest of the section is devoted to the proof of Proposition 1. We first prove

Lemma 9. If $\beta_{q}<\infty$ with $q>0$ then the kernels $\mathbf{Q}$ and $\widetilde{\mathbf{Q}}$ belong to $\mathcal{H}_{1}\left(W, \beta_{q}, 1\right)$ with $W(x) \equiv e^{q x}$.

Proof. It follows from Proposition 5.4 of [4] that

$$
\mathbf{Q}(\cdot \mid \cdot) \in \mathcal{H}_{1}\left(W, \beta, W(0), \boldsymbol{\nu}_{0}, h\right) \quad \text { and } \quad \widetilde{\mathbf{Q}}(\cdot \mid \cdot) \in \mathcal{H}_{1}\left(W, \widetilde{\beta}, W(0), \boldsymbol{\nu}_{0}, \widetilde{h}\right)
$$

where $\boldsymbol{\nu}_{0}$ is the Dirac measure concentrated at $x=0$,

$$
\begin{gathered}
h(x):=\mathbf{P}(x+\zeta \leq 0), \quad \widetilde{h}(x):=\mathbf{P}(x+\widetilde{\zeta} \leq 0), \\
\beta=\mathbf{E} e^{q \zeta} \leq \beta_{q}, \quad \widetilde{\beta}=\mathbf{E} e^{q \widetilde{\zeta}} \leq \beta_{q}, \quad W(x)=e^{q x} .
\end{gathered}
$$

This fact implies the assertion of Lemma 9. 
LEMma 10. If $W(x) \equiv e^{q x}$ for some $q>0$ then

$$
\left\|\varrho_{W, 1}\right\|_{W}:=\sup _{x \geq 0} \frac{\varrho_{W, 1}(x)}{e^{q x}} \leq r_{q} .
$$

Proof. Assume that the distributions of the random variables $\zeta$ and $\widetilde{\zeta}$ have densities $g$ and $\widetilde{g}$, respectively, with respect to some measure $\boldsymbol{\mu}$. (This assumption does not restrict the generality, because we may everywhere put $\boldsymbol{\mu}(B)=\mathbf{P}(\zeta \in B)+\mathbf{P}(\widetilde{\zeta} \in B)$.) It now follows from the definitions (6) and (68) that (see also $[4$, p. 235])

$$
\varrho_{W, 1}(x)=\int e^{q y}\left|d \mathbf{P}\left((x+\zeta)^{+} \leq y\right)-d \mathbf{P}\left((x+\widetilde{\zeta})^{+} \leq y\right)\right| \equiv J_{0}+J_{\infty}
$$

where

$$
\begin{aligned}
J_{0} & =\left|\mathbf{P}\left((x+\zeta)^{+}=0\right)-\mathbf{P}\left((x+\widetilde{\zeta})^{+}=0\right)\right|=|\mathbf{P}(\zeta \leq-x)-\mathbf{P}(\widetilde{\zeta} \leq-x)| \\
& \leq \int_{t \leq-x}|g(t)-\widetilde{g}(t)| \boldsymbol{\mu}(d t) \leq e^{q x} \int_{y \leq-x}|g(t)-\widetilde{g}(t)| \boldsymbol{\mu}(d t),
\end{aligned}
$$

and

$$
\begin{aligned}
J_{\infty} & =\int_{y>0} e^{q y}|d \mathbf{P}(x+\zeta \leq y)-d \mathbf{P}(x+\widetilde{\zeta} \leq y)| \\
& =\int_{t>-x} e^{q(x+t)}|d \mathbf{P}(\zeta \leq t)-d \mathbf{P}(\widetilde{\zeta} \leq t)| \\
& =e^{q x} \int_{t>-x} e^{q t}|g(t)-\widetilde{g}(t)| \boldsymbol{\mu}(d t) \\
& \leq e^{q x} \int_{t>-x} \max \left\{e^{q t}, 1\right\}|g(t)-\widetilde{g}(t)| \boldsymbol{\mu}(d t) .
\end{aligned}
$$

Thus, from (75)-(77) we have

$$
\varrho_{W, 1}(x) \leq e^{q x} \int_{-\infty}^{\infty} \max \left\{e^{q t}, 1\right\}|g(t)-\widetilde{g}(t)| \boldsymbol{\mu}(d t) \equiv e^{q x} r_{q} .
$$

The latter equality follows from (72) and implies the desired inequality (74).

Now, the assertion of the proposition follows immediately from Corollary 2 and Lemmas 9 and 10.

Acknowledgements. The authors would like to thank the three referees for comments and suggestions that improved the paper.

\section{References}

[1] N. M. van Dijk, Perturbation theory for unbounded Markov reward processes with applications to queueing, Adv. Appl. Probab. 20 (1988), 99-111. 
[2] N. M. van Dijk and M. L. Puterman, Perturbation theory for Markov reward processes with applications to queueing systems, ibid. 20 (1988), 79-98.

[3] E. Gordienko, An estimate of the stability of optimal control of certain stochastic and deterministic systems, J. Soviet Math. 50 (1992), 891-899. (Translated from the Russian publication of 1989.)

[4] E. Gordienko and O. Hernández-Lerma, Average cost Markov control processes with weighted norms: value iteration, Appl. Math. (Warsaw) 23 (1995), 219-237.

[5] E. Gordienko and F. Salem-Silva, Robustness inequality for Markov control processes with unbounded costs, Systems Control Lett. 33 (1998), 125-130.

[6] -, - Estimates of Markov control processes with unbounded costs, Kybernetika 36 (2000), 2, 195-210.

[7] O. Hernández-Lerma and J. B. Lasserre, Further Topics on Discrete-Time Markov Control Processes, Springer, New York, 1999.

[8] N. V. Kartashov, Strong stable Markov chains, J. Soviet Math. 34 (1986), 1493-1498.

[9] G. O. Roberts, J. S. Rosenthal and P. O. Schwartz, Convergence properties of perturbed Markov chains, J. Appl. Probab. 35 (1998), 1-11.

[10] J. S. Rosenthal, Minorization conditions and convergence rates for Markov chain Monte Carlo, J. Amer. Statist. Assoc. 90 (1995), 558-566.

[11] W. Whitt, Approximations of dynamic programs, J. Math. Oper. Res. 3 (1978), $231-243$.

Departamento de Matemáticas

Universidad Autónoma

Metropolitana-Iztapalapa

Av. San Rafael Atlixco 186, Col. Vicentina

México D.F. 09340, Mexico

E-mail: momr@xanum.uam.mx
Facultad de Ciencias Físico Matemáticas Benemérita Universidad Autónoma de Puebla Av. San Claudio y Río Verde Col. San Manuel, Ciudad Universitaria Puebla Pue. 72570, Mexico E-mail: fsalem@fcfm.buap.mx

Received on 2.5.2002;

revised version on 23.8.2002 